\title{
Physical Chemistry Chemical Physics
}

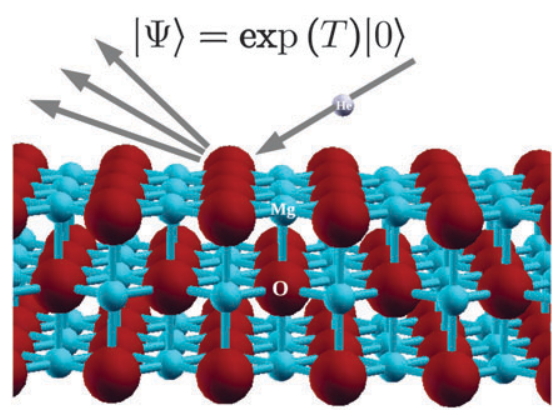

Diffraction of helium on $\mathrm{MgO}(100)$ surface calculated from first-principles

Ruth Martinez-Casado, * Giuseppe Mallia, Denis Usvyat,* Martin Schütz, Lorenzo Maschio, Silvia Casassa, John Ellis and Nicholas M. Harrison

In this work we simulate the diffraction peak intensities of He beams scattered on the $\mathrm{MgO}(100)$ surface from first principles.

Please check this proof carefully. Our staff will not read it in detail after you have returned it.

Translation errors between word-processor files and typesetting systems can occur so the whole proof needs to be read. Please pay particular attention to: tabulated material; equations; numerical data; figures and graphics; and references. If you have not already indicated the corresponding author(s) please mark their name(s) with an asterisk. Please e-mail a list of corrections or the PDF with electronic notes attached - do not change the text within the PDF file or send a revised manuscript. Corrections at this stage should be minor and not involve extensive changes. All corrections must be sent at the same time.

Please bear in mind that minor layout improvements, e.g. in line breaking, table widths and graphic placement, are routinely applied to the final version.

Please note that, in the typefaces we use, an italic vee looks like this: $v$, and a Greek nu looks like this: $\nu$.

We will publish articles on the web as soon as possible after receiving your corrections; no late corrections will be made.

Please return your final corrections, where possible within $\mathbf{4 8}$ hours of receipt, by e-mail to: pccp@rsc.org 


\section{Queries for the attention of the authors}

Journal: PCCP

Paper: c4cp01145g

Title: Diffraction of helium on $\mathrm{MgO}(100)$ surface calculated from first-principles

Editor's queries are marked on your proof like this $Q 1, Q_{2}$, etc. and for your convenience line numbers are indicated like this $5,10,15, \ldots$

Please ensure that all queries are answered when returning your proof corrections so that publication of your article is not delayed.

\begin{tabular}{|l|l|l|}
\hline $\begin{array}{c}\text { Query } \\
\text { reference }\end{array}$ & \multicolumn{1}{c|}{ Query } & Remarks \\
\hline Q1 & $\begin{array}{l}\text { For your information: You can cite this article before you } \\
\text { receive notification of the page numbers by using the } \\
\text { following format: (authors), Phys. Chem. Chem. Phys., } \\
\text { (year), DOI: 10.1039/c4cp01145g. }\end{array}$ & \\
\hline Q2 & $\begin{array}{l}\text { Please carefully check the spelling of all author names. } \\
\text { This is important for the correct indexing and future } \\
\text { citation of your article. No late corrections can be made. }\end{array}$ & \\
\hline Q3 & Please check that the inserted GA text is suitable. & \\
\hline Q4 & $\begin{array}{l}\text { Ref. 21: Can this reference be updated? Please supply } \\
\text { details to allow readers to access the reference (for } \\
\text { references where page numbers are not yet known, } \\
\text { please supply the DOI). }\end{array}$ & \\
\hline Q5 & Please provide the document number for ref. 40. & \\
\hline Q6 & Ref. 50: Please provide the page (or article) number(s). & \\
\hline
\end{tabular}




\title{
Diffraction of helium on $\mathrm{MgO}(100)$ surface calculated from first-principles $\dagger$
}

\author{
Ruth Martinez-Casado, ${ }^{* a}$ Giuseppe Mallia, ${ }^{a}$ Denis Usvyat, ${ }^{* b}$ Martin Schütz, \\ Lorenzo Maschio, ${ }^{c}$ Silvia Casassa, ${ }^{a c}{ }^{2}$ John Ellis ${ }^{d}$ and Nicholas M. Harrison ${ }^{a e}$
}

Received 17th March 2014, Accepted 1st May 2014

DOI: $10.1039 / c 4 c p 01145 g$
In this work we simulate the diffraction peak intensities of He beams scattered on the $\mathrm{MgO}(100)$ surface from first principles. It turns out that diffraction peak intensities are extremely sensitive to the quality of the potential describing the $\mathrm{He}-\mathrm{MgO}$ surface interaction. Achieving the required accuracy in first principles calculations is very challenging indeed. The present work describes a first principles protocol able to achieve very high accuracy for reasonable computational cost. This method is based on periodic local second-order Møller-Plesset where systematic corrections for basis set truncation and for electronic correlation are introduced using coupled cluster calculations on finite model systems mimicking the target system. For the $\mathrm{He}-\mathrm{MgO}$ system the requirements with respect to the level of theory are very high; it turns out that contributions from connected quadruple excitations are non-negligible. Here we demonstrate that using this protocol, it is possible to reach the accuracy in the He-MgO potential that is required to predict the observed He diffraction peak intensities.

\section{Introduction} great fundamental and technological interest and extensively studied both experimentally and theoretically. The scattering of He atom beams on crystal surfaces has the potential to be an important technique for determining the atomistic structure and dynamics of surfaces. ${ }^{1-6}$ The He beam scatters from only the outermost surface layers unlike X-ray diffraction and it neither damages or charges the surface unlike electron diffraction and microscopy. During the last two decades its usefulness has been demonstrated in the determination of numerous surface structures. ${ }^{6-11}$ The quantitative interpretation of $\mathrm{He}-$ diffraction is, however, limited as the He-surface interaction potential is not known accurately. An accurate calculation of the $\mathrm{He}$-surface interaction requires a quantitative description of both short range repulsive forces dominated by electronic exchange and Coulomb interactions and of the long range van

\footnotetext{
${ }^{a}$ Thomas Young Centre, Department of Chemistry, Imperial College London, South Kensington London SW7 2AZ, UK. E-mail: r.martinezcasado@imperial.ac.uk

${ }^{b}$ Institut für Physikalische und Theoretische Chemie, Universität Regensburg, Universitätsstrasse 31, 93040 Regensburg, Germany.

E-mail: denis.usvyat@chemie.uni-regensburg.de

${ }^{c}$ Dipartimento di Chimica, Università degli Studi di Torino, I-10125 Turin, Italy

${ }^{d}$ Cavendish Laboratory, University of Cambridge, JJ Thomson Avenue, Cambridge CB3 OHE, UK

${ }^{e}$ Daresbury Laboratory, Daresbury, Warrington, WA4 4AD, UK

$55 \dagger$ Electronic supplementary information (ESI) available. See DOI: 10.1039/ с4ср01145g
}

der Waals interaction due to electronic correlation. The long range part of the interaction is governed by the slowly decaying van der Waals dispersion, which is weak and very difficult to calculate with high quantitative accuracy. The short range part, on the other hand, is dominated by the exponentially growing repulsive wall originating from the exchange-interaction between the (mutually penetrating) electron distributions of surface and $\mathrm{He}$ atom. The functional form of the interaction potential can be conveniently summarised by the corrugation function, $z(E, x, y)$, which is an isovalue surface of the energy of interaction, $E(x, y, z)$, at the kinetic energy of the He atom beam.

The positions of the He-diffraction peaks ("channels") are determined by the kinetic energy and direction of the He-beam and the periodicity of the corrugation function which is determined by the surface unit cell. The variation of the intensity in each channel with the kinetic energy of the helium beam is determined by the detailed shape of $z(E, x, y) \cdot{ }^{3,6,12}$ At first sight it would seem that diffraction is solely governed by the shortrange repulsive interactions, but in fact the van der Waals dispersion lowers the energy of the repulsive wall and thus influences the diffraction process quite substantially. As will be shown below, the sensitivity of the relative intensities of the helium beams, diffracted in various channels, to the corrugation amplitude is extremely strong. For instance, a variation of the latter by $10 \%$ can change the relative intensity by a factor of 2. Therefore, in order to predict from first principles the relative intensities of helium in different diffraction channels, a method for calculating the interaction potential with a precision of a few percent is required. For simple systems with a few atoms in the 
1 surface unit cell, empirical interaction potentials based on pairwise atomic interactions can be used to fit the diffraction pattern for a range of energies. Yet in order to unambiguously determine the surface structure from the diffraction pattern an independent and reliable determination of the He-surface interaction is required. In systems with complex surface reconstructions or multiple atoms in the surface unit cell, fitting the pairwise potential to experimental data is insufficient for unambiguous structural determination.

10 A reliable calculation of the interaction potential is non trivial. Density functional theory is very widely used to determine surface adsorption energetics ${ }^{6}$ but is unreliable for describing nonbonded and long range van der Walls interactions. DFT benchmark calculations demonstrate this point clearly; there is a very strong variation with the details of the exchange correlation functional adopted. ${ }^{13}$ Empirical models ${ }^{14,15}$ for the energy surface depend on ad hoc parameters, which undermines the predictive value of the calculations.

The quantum chemical hierarchy of theoretical models, on the other hand, allows for the treatment of electron correlation at an increasing level of theory and thus a systematic increase in the accuracy of the computed energy surface. For He-MgO surface interactions the bottom rung of the hierarchy, second-order Møller-Plesset perturbation theory (MP2), has been recently demonstrated to provide a qualitatively correct but quantitatively inadequate description of such systems. ${ }^{16-18}$ As will be demonstrated below it is necessary to include up to connected quadruple excitations in the many body wavefunction (e.g. by means of the coupled cluster singles, doubles, triples and perturbative quadruples [CCSDT(Q)] method $^{19}$ ) in order to compute a reliable energy surface.

In this work we apply a periodic/finite cluster hierarchical scheme to calculate the interaction potential between helium and the $\mathrm{MgO}(100)$ surface. It consists of the periodic local MP2 treatment, which is subsequently corrected on the basis of finite cluster calculations, in order to achieve an energy surface at the $\operatorname{CCSD}(\mathrm{T}) /$ extrapolated-basis-set and CCSDT(Q) levels of theory. Similar computational protocols have already been applied in earlier work to study the geometrical frustration of an argon monolayer adsorbed on $\mathrm{MgO}(100)^{20}$ and to determine the lowest 0 bound state of helium adsorbed on $\mathrm{MgO}(100) .{ }^{21}$ Scattering simulations are then performed for various energies, polar angles, and two different azimuthal angles of the incident beam, for which high quality experimental scattering data is available., ${ }^{7,22}$

The paper is organized as follows. In Sections IIA and IIB we

45 present the description of the computational schemes, used to calculate the $\mathrm{He}-\mathrm{MgO}$ potential surface and the diffraction intensities, respectively. The results of the calculations and the related discussion is given is Section III. Finally Section IV concludes the paper.

\section{Computational methods}

\section{A. He-MgO interaction potential}

The hierarchy of molecular ab initio electronic structure methods allows it in principle to reach very high precision in describing intermolecular interactions. However, the steep scaling of the computational cost with molecular size prevents the use of highorder canonical coupled cluster methods already for moderately large systems. This problem can be circumvented by instead using local coupled cluster methods ${ }^{23,24}$ or DFT-based symmetry adapted perturbation theory (SAPT). ${ }^{25,26}$ The latter has the additional bonus of naturally partitioning the interaction energy into physically meaningful components, and therefore provides detailed information on the individual components of the interaction potential.

In the case of surface adsorption, the surface is effectively infinite in extent, and a purely molecular treatment becomes impossible unless an appropriate embedding is set up to mimic the influence of the environment on the cluster. ${ }^{27}$ Unfortunately, due to the delicacy of the interaction between the adsorbate and the surface, such an embedding has to be tuned with great care, since otherwise artefacts, noticeable at the scale of interaction energies, compromise the interaction potential. ${ }^{28}$ It is much safer to use finite clusters not to compute the full interaction energy, but only to calculate the corrections to the periodic result, obtained e.g. at the periodic $\mathrm{HF},{ }^{29,30}$ DFT-D, ${ }^{31,32}$ or even at the MP2 levels ${ }^{20}$ (the smaller the corrections the better). This becomes especially important for the description of the scattering of helium atoms at a surface, where a highly accurate corrugation function $z(E, x, y)$, and thus a very precise interaction potential, is essential. To this end we employ the highest-order correlation treatment available in the periodic atomic-orbital (AO) format, which presently is the periodic local MP2 (LMP2) method, ${ }^{16-18}$ and compute high-order corrections (up to CCSDT(Q)) based on finite cluster calculations on top of that platform.

An accurate correlated description also requires rather large basis sets. ${ }^{33}$ In order to capture dispersion the basis set has to be augmented with diffuse AOs. Yet the quality of the basis set in periodic calculations is somewhat limited, since the overlap between AOs can cause numerical instabilities, particularly so for rich basis sets including diffuse functions. For the periodic LMP2 calculations thus a decent basis set was employed, i.e., of triple-zeta quality for $\mathrm{Mg}$ and $\mathrm{O}$, and of quadruple-zeta quality for $\mathrm{He}$, augmented with diffuse $\mathrm{p}$ - and f-functions for $\mathrm{O}$, and diffuse s-, p-, d- and f-functions for He. The Mg cc-pVTZ basis set initially already contains very diffuse functions, which cannot be used in the periodic context due to above mentioned redundancies. Such functions were either omitted or upscaled. The detailed specification of the employed basis set can be found in ref. 34. For further reference we denote it as AVTZ. This basis is sufficiently large to reach saturation at the Hartree-Fock (HF) level, ${ }^{21}$ but not yet for the correlation energy contribution. Hence, the remaining basis set incompleteness error is corrected together with above mentioned method error correction scheme, as described in detail below.

The periodic LMP2 potential energy surface (PES) was calculated as the interaction energy per helium atom between an $\mathrm{MgO}(100)$ 3-layer slab (with a experimental lattice parameter of $4.211 \AA$ ) and a square monolayer of $\mathrm{He}$ matching the surface lattice of $\mathrm{MgO}$ (for the corresponding $\mathrm{He}-\mathrm{He}$ distances the He-He interaction is negligible). Rumpling of the surface, which is observed 
1 experimentally $^{35}$ for $\mathrm{MgO}(100)$, is not included in the LMP2 calculations. Yet, its influence on the diffraction intensities is explored at a later stage (see Section III). The inter-He-slab correlation interaction (dispersion), evaluated explicitly for the 3 layer slab, was then extrapolated to the semi-infinite crystal limit using the slab replication technique. ${ }^{18}$ The He-MgO PES was represented by a uniform grid consisting of 313 points. All calculations have been performed using the CRYSTAL09 ${ }^{36}$ and CRYSCOR09 ${ }^{18}$ software packages, both based on the expansion

10 of the crystalline orbitals as a linear combination of a local basis set (BS) consisting of atom centred Gaussian orbitals (see ref. 13 for details). The potential curves for 21 symmetry unique adsorption sites were calculated, each sampled by 14-17 points lying in the range of 2 to $7 \AA$ along the He-surface direction $z$.

Since the valence electrons in $\mathrm{MgO}$ are localized around the oxygen atoms the interaction between $\mathrm{He}$ and $\mathrm{MgO}$ is mainly a competition of dispersive attraction and exchange repulsion between the electron clouds around oxygen and those of helium. The contribution of the upper core electrons of $\mathrm{Mg}$ to dispersion is small, but still non-negligible at the scale of the interaction energy. ${ }^{20,31,32}$ In order to take it into account, additional frozen-core and correlated 2sp-Mg-core periodic LMP2 calculations were performed for two different adsorption sites. The core contribution was evaluated as the difference between the interaction energies of these two calculations. For adjusting the basis set to the describe core correlation effects, additional tight AOs from cc-pwCVTZ ${ }^{37}$ (s- and p-functions) and cc-pCVTZ (d- and f-functions) basis sets were added on the $\mathrm{Mg}$ atoms. Since the core correlation calculations were already quite expensive, the core contributions were explicitly evaluated for the on-Mg and on-oxygen adsorption sites only, i.e., the most attractive and repulsive adsorption sites on the surface. It turned that the core correlation contribution is rather isotropic along in-plane directions. Hence, in order to extrapolate the core correlation contribution for an arbitrary site we applied for it the $2 \mathrm{D}$-sine law model. ${ }^{20}$

It has recently been established, ${ }^{20,21,34}$ that the MP2 level of theory significantly underestimates the adsorption energy of noble gasses on the MgO surface, and especially so for He.

40 Moreover, this underestimation is further magnified by the basis set deficiency error, which has the same sign as the MP2 method error for these systems. The latter problem can be eliminated to large extend by the recently implemented periodic LMP2-F12 method, ${ }^{38}$ which unfortunately is yet computape to be used for the whole $\mathrm{He}-\mathrm{MoO}$ potential surface. Therefore, we employ a recently developed correction scheme, ${ }^{20}$ where method and basis set deficiencies are corrected on the basis of finite cluster calculations. In this scheme, the periodic LMP2 result is corrected by scaling the intra-slab $\Delta E_{\text {intra-MgO }}^{\mathrm{LMP2}}$ and inter-adsorbate-slab $\Delta E_{\text {inter }}^{\mathrm{LMP} 2}$ components of the correlation part of the interaction energy ${ }^{18,39}$ with appropriate factors $f_{\text {intra-Mgo }}$ and $f_{\text {inter }}$, respectively. In contrast to other common correction schemes based on universal or empirical parameters (like e.g. the popular spin-component-scaled MP2), here the scaling factors are determined by comparing the $\operatorname{CCSD}(\mathrm{T}) /$ basis-set-extrapolated treatment to the LMP2 treatment (in the basis of the periodic calculation) for a specific finite model system characterized by the same kind of interactions as the actual system of interest. In comparison to formally more rigorous approaches, where finite-cluster corrections are added to the periodic interaction energies in the form of energy increments, ${ }^{31,32}$ our scheme is computationally more advantageous, since the whole LMP2 $\mathrm{He}-\mathrm{MgO}$ potential surface is corrected in a single step.

Similar to previous work, ${ }^{21}$ we employed the $\mathrm{He}-\mathrm{Mg}_{3} \mathrm{Na}_{2} \mathrm{O}_{4}$ dimer (see ESI, ref. 40) as the finite model system mimicking the periodic $\mathrm{He}-\mathrm{MgO}$ system. A system of this size still allows for a $\operatorname{CCSD}(\mathrm{T})$ treatment with augmented basis sets of up to quadruple-zeta quality (and thus a reasonably accurate extrapolation to the basis set limit). Two sodium atoms (yet equipped with $\mathrm{Mg}$ instead of $\mathrm{Na}$ basis functions) replace the $\mathrm{Mg}$ atoms at opposite corners of the cluster to keep the charge-neutrality and the ionic type of binding in this cluster. Pure $\mathrm{MgO}$ clusters (without sodium atoms) of similar size, such as the flat $\mathrm{Mg}_{6} \mathrm{O}_{6}$ cluster or a $\mathrm{Mg}_{5} \mathrm{O}_{5}$ cluster with one oxygen atom below the central $\mathrm{Mg}$, unfortunately have multireference character, and therefore cannot be used for evaluating the correction factors. A brick-like $\mathrm{Mg}_{9} \mathrm{O}_{9}$ cluster, on the other hand, is not a multireference case, but yet too large for the quadruple-zeta $\operatorname{CCSD}(\mathrm{T})$ treatment.

The $\mathrm{He}-\mathrm{Mg}_{3} \mathrm{Na}_{2} \mathrm{O}_{4}$ dimer was subjected to frozen-core local MP2 and $\operatorname{CCSD}(\mathrm{T})$ treatments using the Molpro program. ${ }^{41-43}$ To correct for the basis set deficiency together with the method error, the same basis set and domains as in the actual periodic LMP2 treatment were also used in the finite-cluster LMP2 calculations. The upscaling factors were determined by fitting the upscaled LMP2 correlation contributions of the respective interaction energies to the $\operatorname{CCSD}(\mathrm{T})$ reference values. To this end we minimize the function

$$
\begin{aligned}
& \sum_{z}\left(\Delta E^{\mathrm{CCSD}(\mathrm{T})}-\Delta E_{\mathrm{intra}-\mathrm{He}}^{\mathrm{LMP}}\right. \\
& \left.-f_{\text {intra- }-\mathrm{Mg}_{3} \mathrm{Na}_{2} \mathrm{O}_{4}} \Delta E_{\text {intra- }}^{\mathrm{LMP} 2} \mathrm{Mg}_{3} \mathrm{Na}_{2} \mathrm{O}_{4}-f_{\text {inter }} \Delta E_{\text {inter }}^{\mathrm{LMP} 2}\right)^{2},
\end{aligned}
$$

where $\Delta E^{\mathrm{CCSD}(\mathrm{T})}, \Delta E_{\text {intra-He }}^{\mathrm{LMP} 2} \Delta E_{\text {intra- } \mathrm{Mg}_{3} \mathrm{Na}_{2} \mathrm{O}_{4}}^{\mathrm{LMP}}$, and $\Delta E_{\text {inter }}^{\mathrm{LMP} 2}$ are the correlation contributions of, respectively, the (basis set extrapolated) $\operatorname{CCSD}(\mathrm{T})$ interaction energy, and the LMP2 intra-He, intra- $\mathrm{Mg}_{3} \mathrm{Na}_{2} \mathrm{O}_{4}$, and $\mathrm{He}-\mathrm{Mg}_{3} \mathrm{Na}_{2} \mathrm{O}_{4}$ inter-monomer energies, all at a certain distance $z$. For the summation (1) $17 z$-values in the range from 2.4 to $7 \AA$ were used. Fig. 1 shows that the LMP2 potential curve for the $\mathrm{He}-\mathrm{Mg}_{3} \mathrm{Na}_{2} \mathrm{O}_{4}$ interaction is indeed far too shallow, yet after upscaling of the intra- and inter-monomer correlation components the $\operatorname{CCSD}(\mathrm{T})$ reference curve is faithfully reproduced by LMP2 over the whole range of relevant inter-monomer distances.

The upscaling parameters corresponding to the $\operatorname{CCSD}(\mathrm{T}) /$ aug-cc-pVTZ (Ups1), CCSD(T)/aug-cc-pVQZ (Ups2) and $\operatorname{CCSD}(\mathrm{T}) /$ aug-cc-pV(TQ)Z-extrapolated (Ups3) are given in Table 1. The inter-monomer correction factor of 1.7 , which reflects the considerable underestimation of dispersion by MP2, is virtually unaffected by the basis set employed in the $\operatorname{CCSD}(\mathrm{T})$ calculation. The intra- $\mathrm{Mg}_{3} \mathrm{Na}_{2} \mathrm{O}_{4}$ correction, on the 


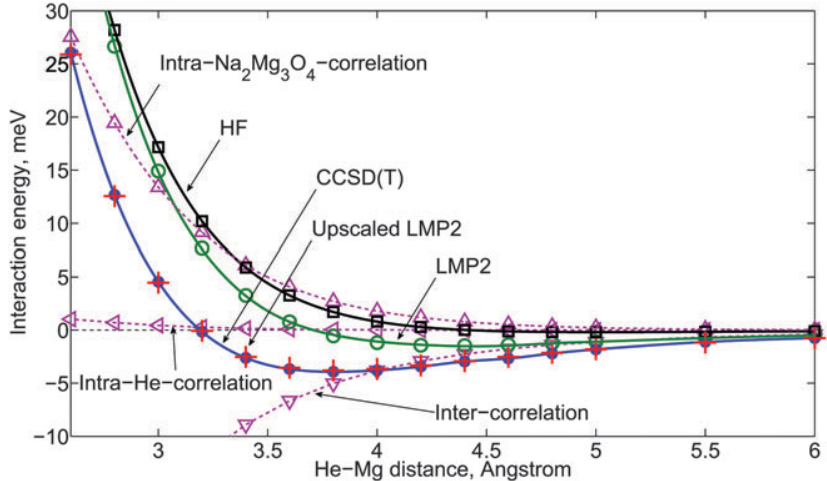

Fig. 1 Interaction energy of the $\mathrm{He}-\mathrm{Mg}_{3} \mathrm{Na}_{2} \mathrm{O}_{4}$ dimer at the Hartree-Fock (black squares), LMP2/AVTZ (green circles) and $\operatorname{CCSD}(T) / a u g-c c-p V(T Q) Z-$ extrapolated (blue small filled circles) levels. The LMP2 correlation interaction energy is partitioned (magenta dashed lines) into inter-component (downpointing triangles), intra- $\mathrm{Mg}_{3} \mathrm{Na}_{2} \mathrm{O}_{4}$-component (up-pointing triangles) and intra-He-component (left-pointing triangles). The LMP2 energies with the scaled intra- $\mathrm{Mg}_{3} \mathrm{Na}_{2} \mathrm{O}_{4}$ - (factor 1.07) and inter- (factor 1.71) components are denoted with red crosses.

Table 1 The scaling factors for the intra- and inter-monomer components of the LMP2/AVTZ correlation contributions to the interaction energy for the $\mathrm{He}-\mathrm{Mg}_{3} \mathrm{Na}_{2} \mathrm{O}_{4}$ system, obtained by fitting to the $\mathrm{CCSD}(\mathrm{T})$ potential curve, calculated with aug-cc-pVTZ (Ups1), aug-cc-pVQZ (Ups2) basis sets, and aug-cc-pV(TQ)Z-extrapolated to the basis set limit (Ups3)

\begin{tabular}{llll}
\hline & Ups1 & Ups2 & Ups3 \\
\hline$f_{\text {intra- } \mathrm{Mg}_{3} \mathrm{Na}_{2} \mathrm{O}_{4}}$ & 1.21 & 1.13 & 1.07 \\
$f_{\text {inter }}$ & 1.67 & 1.69 & 1.71
\end{tabular}

other hand, is sensitive to the basis set. Interestingly, the quite moderate value of 1.07 for the intra-monomer correction factor indicates that in this particular component the method error of LMP2 and the basis set incompleteness error (of the AVTZ basis as used in the periodic calculation) compensate each other to a large extent when referenced to the $\operatorname{CCSD}(\mathrm{T}) /$ basis-set-extrapolated value. However, as was recently demonstrated ${ }^{21}$ for a single point CCSDT(Q) calculation on a small $\mathrm{He}-\mathrm{Mg}_{2} \mathrm{O}_{2}$ cluster, the $\operatorname{CCSD}(\mathrm{T})$ method is not yet a converged reference for this system with respect to the order of the correlated method (at least at the scale of the interaction energies). Therefore the upscaling factors corresponding to the $\operatorname{CCSD}(\mathrm{T}) /$ basis-set-extrapolated results do not provide sufficient accuracy. As will be shown below, the relative diffraction intensities manifest a high sensitivity to deviations in the corruga$\operatorname{CCSD}(\mathrm{T}) /$ basis-set-extrapolated description lead to significant errors in the intensities. Hence, a higher order correlated description becomes mandatory for reaching reasonable accuracy in the diffraction intensities. This also rules out the use of the DFT-SAPT method, ${ }^{25,26}$ which is usually quite accurate for the inter-molecular interactions, but still inferior to $\operatorname{CCSD}(\mathrm{T})$, and hence definitely not sufficiently accurate for the present system.

Unfortunately, the $\mathrm{He}-\mathrm{Mg}_{3} \mathrm{Na}_{2} \mathrm{O}_{4}$ cluster is too large for a $\operatorname{CCSDT}(\mathrm{Q})$ treatment, making the direct computation of the correcting factors at the CCSDT(Q) level unfeasible. In order to include the higher order correlation correction in our scheme,

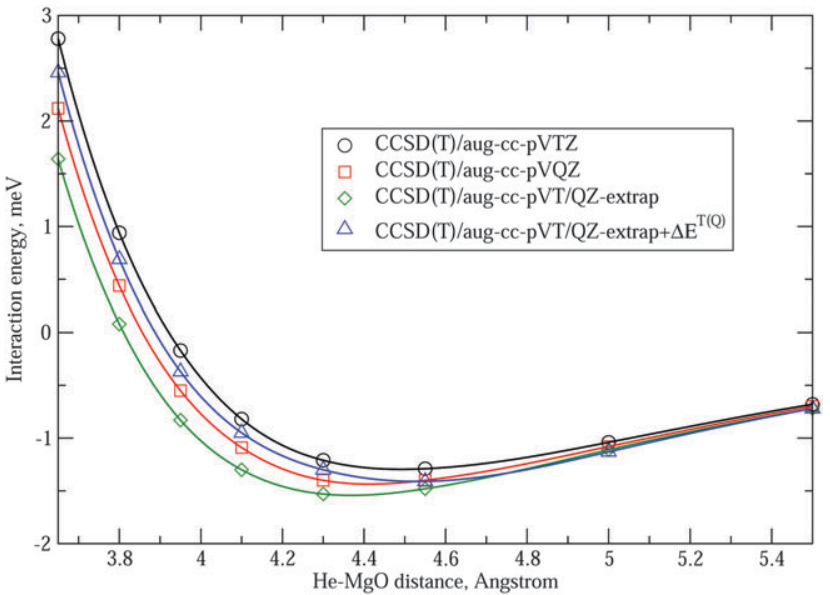

Fig. 2 The $\operatorname{CCSD}(\mathrm{T})$ interaction energies of the $\mathrm{He}-\mathrm{Mg}_{2} \mathrm{O}_{2}$ dimer at the augcc-pVTZ (black circles), aug-cc-pVQZ (red squares) and aug-cc-pV(TQ)Zextrapolated (green diamonds) basis sets levels. The curve resulting from adding the $\operatorname{CCSDT}(\mathrm{Q})-\operatorname{CCSD}(\mathrm{T})$ energy differences (denoted as $\Delta E^{T(Q)}$ ) to the $\operatorname{CCSD}(T) / a u g-c c-p V(T Q) Z$-extrapolated values is also given (blue triangles).

we calculated the $\operatorname{CCSDT}(\mathrm{Q})-\mathrm{CCSD}(\mathrm{T})$ interaction energy difference with a moderate basis set (cc-pVDZ for $\mathrm{Mg}$, aug-cc-pVDZ for $\mathrm{O}$, and aug-cc-pVTZ for $\mathrm{He}$ ) for the $\mathrm{He}-\mathrm{Mg}_{2} \mathrm{O}_{2}$ dimer with $\mathrm{He}$ placed on top of the $\mathrm{Mg} \mid \mathrm{Mg}$-bridge-position (see ESI, ref. 40). For these calculations Kallay's MRCC program, ${ }^{19,44}$ interfaced with Molpro, was used. This energy increment was added to the $\operatorname{CCSD}(\mathrm{T}) / \mathrm{basis}$ set extrapolated result for the same cluster. It is assumed here that the basis set effects for the $\operatorname{CCSDT}(\mathrm{Q})$ $\operatorname{CCSD}(\mathrm{T})$ energy correction, which is of the fifth-order within the MP partitioning, are much less important than for the correlation energy itself. ${ }^{45}$ It is evident from Fig. 2 that in the long range regime the $\operatorname{CCSDT}(\mathrm{Q})-\operatorname{CCSD}(\mathrm{T})$ correction is virtually zero, whereas in the short range regime, i.e. in the repulsive wall region it is substantial. Furthermore, the $\operatorname{CCSDT}(\mathrm{Q})-\operatorname{CCSD}(\mathrm{T})$ corrected $\operatorname{CCSD}(\mathrm{T}) /$ aug-cc-pVT/QZ-extrapolated curve is sandwiched by the $\operatorname{CCSD}(\mathrm{T}) /$ aug-cc-pVQZ and $\operatorname{CCSD}(\mathrm{T}) /$ aug-cc-pVTZ curves. In previous work, ${ }^{21}$ by performing a single-point periodic LMP2-F12 calculation (to reach the basis set limit directly in the periodic system) and an incremental CCSD(T)-LMP2 energy correction on a larger $\mathrm{He}-\mathrm{Mg}_{9} \mathrm{O}_{9}$ cluster, it was shown that the upscaling factors obtained according to the protocol described above, lead to a slight overestimation of the binding. The upscaling parameters as obtained for the CCSD(T)/aug-cc-pVTZ curve (i.e. Ups1 of Table 1) are therefore anticipated to provide the most accurate diffraction intensities.

\section{B. Diffraction intensities}

The diffraction intensities for various helium beam energies were calculated by solving the helium scattering Schrödinger equation

$$
\left[\nabla^{2}+k_{i}^{2}-\frac{2 \mu}{\hbar^{2}} V(\mathbf{r})\right] \Psi(\mathbf{r})=0
$$

where $k_{i}$ is the incident wave vector, $\mu$ is the mass of the helium atom and $V(\mathbf{r})$ is the Born-Oppenheimer $\mathrm{He}-\mathrm{MgO}$ interaction 
1 potential. Eqn (2) was solved by means of the close-coupling method,,$^{2,3,9,10,14,46-48}$ which implies expansion of the wavefunction $\Psi(\mathbf{r})$ in the plane-wave basis. The surface atoms, on the other hand, are treated classically at zero K surface temperature, i.e., they are motionless. Finite temperature effects can be modeled on the basis of Debye-Waller factors ${ }^{2,9}$ (vide infra). The directions of the diffracted beams are obtained from the two-dimensional Bragg conditions, which depend on the length (energy) and the component parallel to the surface of the wave vector of the incident beam. The detailed formalism of the method can be found for example in ref. 3. All close-coupling calculations were carried out by using the program CPXCC. ${ }^{2,3,46}$

In order to compare the calculated intensities to experimental reference data finite temperature effects have to be ken into account. The thermal correction at a certain surface temperature due to classical vibrations of the surface atoms in the direction perpendicular to the surface is given by the corresponding Debye-Waller factor. ${ }^{2,9}$ It relates the intensity $I(T)$ at that temperature $T$ of a diffraction peak to the zerotemperature intensity $I_{0}$ by

$$
I(T)=I_{0} \mathrm{e}^{-2 W(T)} .
$$

Treating each atom as an independent Einstein oscillator, the dependence of the exponent on the temperature takes the form

$$
2 W(T)=\frac{3 \hbar^{2} T\left(k_{i z}+k_{f z}\right)^{2}}{M k_{\mathrm{B}} \Theta_{\mathrm{D}}^{2}},
$$

where $\Theta_{\mathrm{D}}$ is the Debye temperature, $k_{i z}$ and $k_{f z}$ are the $z$ projections of the incident and diffracted wave-vectors, $M$ is the averaged reduced mass of the surfaces atoms, and $k_{\mathrm{B}}$ the Boltzmann constant. For our system the Debye temperature is $495 \mathrm{~K}^{49}$ and the experiments, ${ }^{7,22}$ used here as the reference, were conducted at temperature $T=300 \mathrm{~K}$. Finally, the acceleration of the helium atoms near the surface due to the attractive potential well with a depth $D$ is taken into account by using the Beeby correction, ${ }^{50}$ which replaces the $z$ components of the initial and final wave vectors with

$$
k_{i / f, z} \Rightarrow \sqrt{k_{i / f, z}^{2}+\frac{2 \mu D}{\hbar^{2}}} .
$$

\section{Calculations}

In order to facilitate the simulation of the diffraction intensities, the potential energy surfaces as obtained from periodic LMP2/Ups1, LMP2/Ups3, and unscaled LMP2 calculations, were

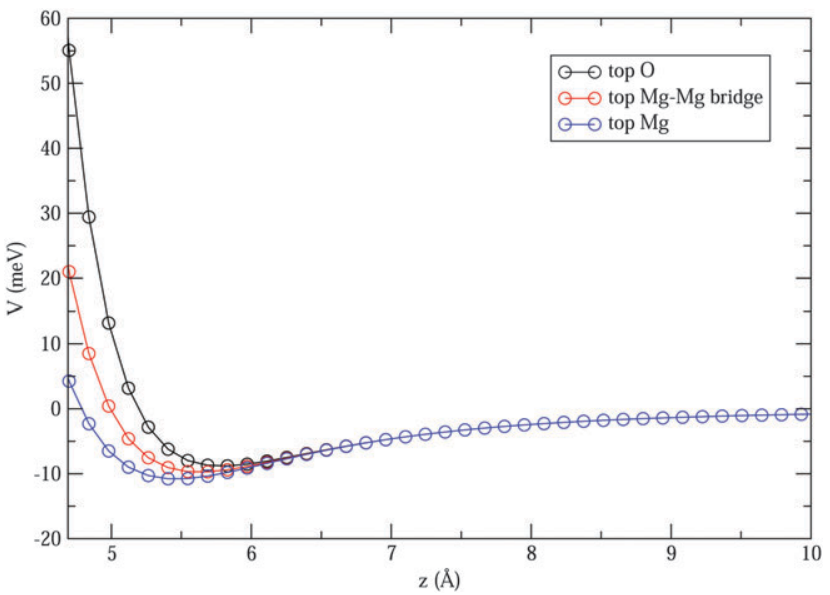

Fig. 3 Fitted potential for various positions of the He on top of the surface: (i) atop $\bigcirc$ (black), (ii) atop $\mathrm{Mg}$ (blue), and (iii) atop the $\mathrm{Mg}-\mathrm{Mg}$ bridge position (red).

subsequently fitted to Buckingham pairwise potentials ${ }^{14}$ by using the program GULP. ${ }^{51}$ The resulting parameters of these model potentials are given in Table 2 .

Fig. 3 displays the LMP2/Ups1 fitted potential along the $z$ direction for three different positions of He atop the surface: (i) atop the oxygen atoms, (ii) atop the Mg-atoms, and (iii) atop the $\mathrm{Mg}-\mathrm{Mg}$ bridge position. The well depths of these potential curves are $-8.80,-10.80$ and $-9.72 \mathrm{meV}$, respectively, while the averaged well depth $D$, employed in eqn (5) is $-9.62 \mathrm{meV}$. In Fig. 3 one can also trace the growth of the amplitude of the corrugation function $z(E, x, y)$ with increasing energy.

To further characterize the LMP2/Ups1 potential we calculated the corresponding energies of the $\Gamma$-point vibrational states. For this purpose a vibrational band-structure planewave program ${ }^{11}$ was used, that solves the Schrödinger eqn (2) for the bound states within the full 3D (2D-periodic) potential. For the LMP2/Ups1 potential 5 bound states were obtained with the energies: $E_{0}=-7.59 \mathrm{meV}, E_{1}=-4.10 \mathrm{meV}, E_{2}=-2.05 \mathrm{meV}$, $E_{3}=-0.85 \mathrm{meV}$, and $E_{4}=-0.30 \mathrm{meV}$, all of them with an error of $\pm 0.01 \mathrm{meV}$. These values are overall in reasonably good agreement with the experimental ones reported in ref. 52 (-5.5 meV, - $2.6 \mathrm{meV},-1.2 \mathrm{meV},-0.5 \mathrm{meV},-0.3 \mathrm{meV})$, especially in the light of the results of ref. 21. As it is argued there, the upscaling model can lead to an overestimation of the well depth by up to $2 \mathrm{meV}$.

Now we focus on the main topic of the current article-the diffraction intensities, which were calculated for various helium beam energies and directions, for which experimental data is available. ${ }^{7}$ The experimental diffraction peak intensities

\begin{tabular}{|c|c|c|c|c|c|c|}
\hline & $A_{\mathrm{HeO}}(\mathrm{meV} \AA)$ & $\rho_{\mathrm{HeO}}(\AA)$ & $C_{\mathrm{HeO}}\left(\mathrm{meV} \AA^{6}\right)$ & $A_{\mathrm{HeMg}}(\mathrm{meV} \AA)$ & $\rho_{\text {HeMg }}(\AA)$ & $C_{\mathrm{HeMg}}\left(\mathrm{meV} \AA^{6}\right)$ \\
\hline LMP2 & $1.4 \times 10^{5}$ & $3.5 \times 10^{-1}$ & $6.3 \times 10^{3}$ & 2.1 & $4.5 \times 10^{-1}$ & $7.0 \times 10^{-1}$ \\
\hline LMP2/Ups1 & $2.2 \times 10^{5}$ & $3.4 \times 10^{-1}$ & $11.7 \times 10^{3}$ & $2.8 \times 10^{1}$ & $4.8 \times 10^{-1}$ & $3.0 \times 10^{-1}$ \\
\hline LMP2/Ups3 & $2.4 \times 10^{5}$ & $3.3 \times 10^{-1}$ & $11.9 \times 10^{3}$ & $4.5 \times 10^{1}$ & $4.8 \times 10^{-1}$ & $2.0 \times 10^{-1}$ \\
\hline
\end{tabular}

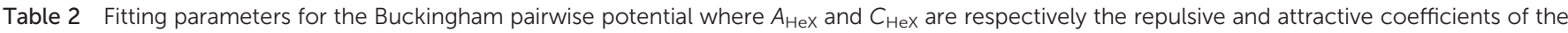
$\mathrm{He}-\mathrm{X}$ interactions (where $\mathrm{X}=\mathrm{O}$ and $\mathrm{Mg}$ ) 
(a)

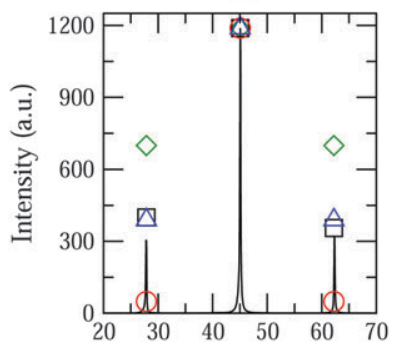

(d)

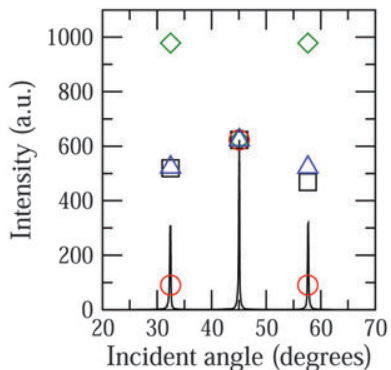

(b)

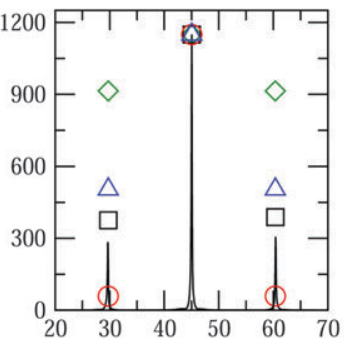

(e)

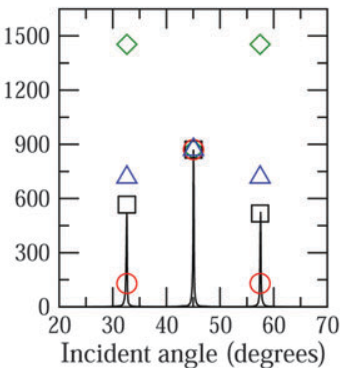

(c)

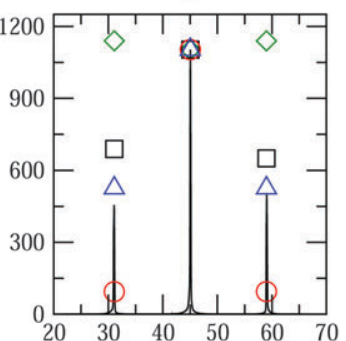

(f)

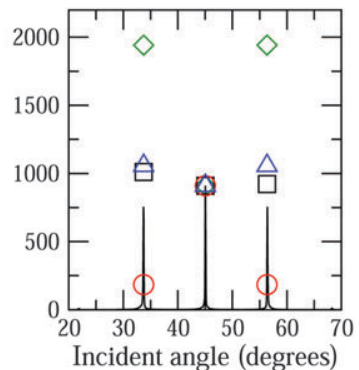

10

Fig. 4 Comparison of the close-coupling intensities at $\langle 100\rangle$ direction of incident beam for the MP2 (red circles), Ups3 (green diamonds) and Ups1 (blue up triangles) with the experimental spectra (black lines) and the peak areas (black squares). The diffraction peaks are given in counts per $\mathrm{s}$; the peak areas in counts $\times$ deg per $\mathrm{s}$, and close-coupling intensities have been normalized in a way that the specular (central) peak appears at the maximum of the experimental peak. The considered incident energy are the following: (a) $E_{i}=26.62 \mathrm{meV}$, (b) $E_{i}=33.30 \mathrm{meV}$, (c) $E_{i}=40.02 \mathrm{meV}$, (d) $E_{i}=48.96 \mathrm{meV}$, (e) $E_{i}=50.20 \mathrm{meV}$ and (f) $E_{i}=60.47 \mathrm{meV}$.

and the corresponding integrated peak areas ${ }^{7,22}$ for the two azimuthal $\langle 100\rangle$ and $\langle 110\rangle$ He-beam directions are displayed in Fig. 4 and 5, respectively. We note that some of the experimental

spectra, presented here, were not explicitly reported in ref. 7, but have been directly provided to us by Dr F. Traeger. It is usually argued (cf. e.g. ref. 53) that the peak areas are a more reliable (a)

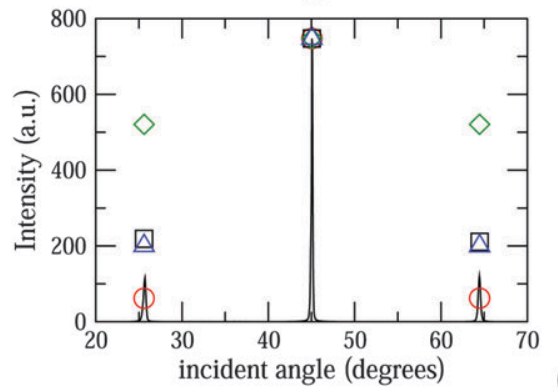

(b)

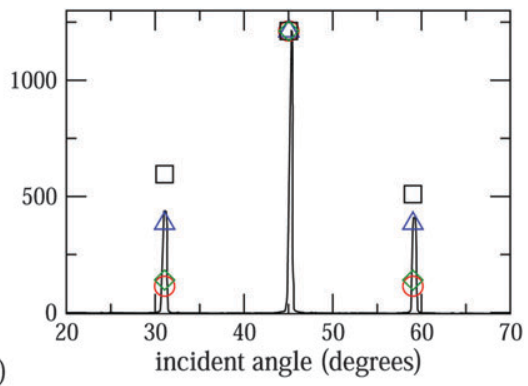

Comparison of the close-coupling intensities at $\langle 110\rangle$ direction of incident beam for the MP2 (red circles), Ups3 (green diamonds) and Ups1 (blue up triangles) with the experimental spectra (black lines) and the peak areas (black squares). The diffraction peaks are given in counts per $\mathrm{s}$; the peak areas in counts $\times$ deg per $\mathrm{s}$, and close-coupling intensities have been normalized in a way that the specular (central) peak appears at the maximum of the experimental peak. The considered incident energy are the following: (a) $E_{i}=10.52 \mathrm{meV}$, (b) $E_{i}=19.93 \mathrm{meV}$, (c) $E_{i}=26.16 \mathrm{meV}$. 
1 representation of the intensity for comparison with calculated intensities, than the peak heights themselves, since effects of diffraction peak broadening due to energy and momentum spread of the He beam, are taken into account in the former.

The same figures also contain the relative intensities (normed to the intensities of the central peaks) calculated by employing the Ups1 model (the most accurate one, $c f$. Section IIA), and according to the Ups3 and pure LMP2 models, for comparison. Agreement of the LMP2 and Ups3 intensities with experiment is clearly very poor, whereas the Ups1 model provides indeed quite accurate diffraction peak intensities. The dramatic improvement of the agreement with experiment for the Ups1 model indicates (i) the sensitivity of the calculated diffraction peak intensities on the quality of the potential energy surface, and (ii) the effectiveness of the correcting 15 scheme described above and the importance of the corrections of both method and basis set deficiencies.

Interestingly, the corrections themselves do not lead to a substantial change in the potential or the corrugation function (e.g. the amplitudes of the corrugation function in the Ups3 and Ups1 models differ only by about $10 \%$ ). However, even such a small modification has extreme consequences for the relative amplitudes: the Ups3 amplitudes deviate from the Ups1 ones by a factor of two. Such a strong sensitivity of the intensities to the form of the surface corrugation renders He scattering as a highly accurate experimental tool to determine He-surface potentials. To reach the required extremely high precision in first-principle calculations of such potentials is indeed very challenging, but possible, as demonstrated by the present work.

Finally, we investigate the influence of the surface rumpling

30 on the intensities. For the $\mathrm{MgO}(100)$ surface, rumpling manifests mainly in a shift of the $\mathrm{Mg}$ atoms inwards by about $0.02 \AA$ (cf. e.g. ref. 35 or ref. 6, and references therein). We recalculated the diffraction patterns employing the fitted LMP2/Ups3 potential for the $\mathrm{MgO}$ with the rumpled surface, but the impact on the intensities was found to be very small. We attribute the low sensitivity of the intensities to rumpling in $\mathrm{MgO}$ to fact that the $\mathrm{He}-\mathrm{MgO}$ interaction (both the attraction and repulsive components thereof) and thus the corrugation function for the low energies involved in this study are mainly determined by the electron clouds around the oxygen atoms, which are not affected significantly by rumpling. At higher energies the effect of the rumpling on the corrugation becomes more pronounced. ${ }^{6}$ Higher energy He beams, accessible via the Fast Atom Diffraction technique, ${ }^{4,5}$ could therefore provide more information about rumpling. ${ }^{6}$

\section{Conclusions}

In this work we have simulated the diffraction peak intensities This is a very challenging endeavor due to the fact that the diffraction peak intensities are very sensitive to the He-surface interaction potential, which in turn poses high demands on the quality of the $a b$ initio electronic structure methods used to construct the potential energy surface. In order to achieve the required precision we applied a recently developed hierarchical first-principle computational protocol to calculate the $\mathrm{He}-\mathrm{MgO}(100)$ interaction energies. It is based on a periodic local MP2 treatment with scaled intra-surface(slab) and inter-surface(slab)-helium correlation components of the interaction energy. The appropriate scaling factors correcting for method- and basis set errors are obtained from LMP2 and CCSD(T)/basis-set-extrapolated calculations on the $\mathrm{He}-\mathrm{Mg}_{3} \mathrm{Na}_{2} \mathrm{O}_{4}$ dimer, which mimics the target system. Actually, as it turns out, even a $\operatorname{CCSD}(\mathrm{T})$ treatment, the "gold standard" of numerical quantum chemistry, provides insufficient accuracy. Hence, we evaluated an additional $\operatorname{CCSDT}(\mathrm{Q})-\operatorname{CCSD}(\mathrm{T})$ correction on the smaller $\mathrm{He}-\mathrm{Mg}_{2} \mathrm{O}_{2}$ dimer (CCSDT(Q) calculations are extremely expensive). The $\operatorname{CCSDT}(\mathrm{Q})-\mathrm{CCSD}(\mathrm{T})$ correction is virtually zero for the long-range part of the $\operatorname{CCSD}(\mathrm{T})$ potential, yet has a noticeable impact on the repulsive part, making it steeper. Generally, it shifts the $\operatorname{CCSD}(\mathrm{T}) /$ basis-set-extrapolated potential curve back to that obtained with $\operatorname{CCSD}(\mathrm{T}) /$ aug-cc-pVTZ. The latter potential thus is expected to provide the most accurate diffraction peak intensities, which were obtained by employing the close-coupling method, with finite temperature effects included via corresponding Debye-Waller factor. The simulated diffraction peak intensities turned out to be in excellent agreement with available experimental data.

We conclude that the proposed methodology opens a way to predict accurate first principles diffraction patterns for helium atoms scattered on non-conducting surfaces. Such calculations will provide important support for experimental investigations of surfaces using helium beam techniques. In future work we plan to apply this method to predict helium diffraction intensities for surfaces of several other oxide crystals.

\section{Acknowledgements}

The authors are grateful to Dr Franziska Traeger for providing some of the experimental results, SC thanks the Thomas Young Centre (TYC) for financial support during a sabbatical visit. In addition, this work made use of the facilities of Imperial College HPC and - via our membership of the UK's HPC Materials Chemistry Consortium funded by EPSRC (EP/F067496) - of HECToR, the UK's national high-performance computing service, which is provided by UoE HPCx Ltd at the University of Edinburgh, Cray Inc and NAG Ltd, and funded by the Office of Science and Technology through EPSRC's High End Computing Programme. DU and MS acknowledge financial support from the Deutsche Forschungsgemeinschaft (Grants US-103/1-1, and SCHU 1456/3-2).

\section{References}

1 S. Miret-Artés, Surf. Sci., 1995, 339, 205.

2 S. Miret-Artés, J. Toennies and G. Witte, Phys. Rev. B: Condens. Matter Mater. Phys., 1996, 54, 5881.

3 A. Sanz and S. Miret-Artés, Phys. Rep., 2006, 451, 37.

4 A. Schüller, S. Wethekam and H. Winter, Phys. Rev. Lett., 2007, 98, 016103.

5 P. Rousseau, H. Khemliche, A. G. Borisov and P. Roncin, Phys. Rev. Lett., 2007, 98, 016104. 
16 A. Schüller, D. Blauth, J. Seifert, M. Busch, H. Winter, K. Gärtner, R. Wlodarczyk, J. Sauer and M. Sierka, Surf. Sci., 2012, 606, 161.

7 G. Benedek, G. Brusdeylins, V. Senz, J. G. Skofronick, J. P. Toennies, F. Traeger and R. Vollmer, Phys. Rev. B: Condens. Matter Mater. Phys., 2001, 64, 125421.

8 J. Gomes and J. P. Ramalho, Phys. Rev. B: Condens. Matter Mater. Phys., 2005, 71, 235421.

9 D. Farias and K.-H. Rieder, Rep. Prog. Phys., 1998, 61, 1575.

10 R. Guantes, A. Sanz, J. Margalef-Roig and S. Miret-Artés, Surf. Sci. Rep., 2004, 53, 99.

11 F. Tuddenham, H. Hedgeland, J. Knowling, A. Jardine, D. MacLaren, G. Alexandrowicz and J. E. W. Allison, J. Phys.: Condens. Matter, 2009, 21, 264004.

12 U. Garibaldi, A. Levi, R. Spadacini and G. Tommei, Surf. Sci., 1975, 48, 649.

13 R. Martinez-Casado, G. Mallia, D. Usvyat, L. Maschio, S. Casassa, M. Schütz and N. M. Harrison, J. Chem. Phys., 2011, 134, 014706.

14 R. Martinez-Casado, G. Mallia, D. Usvyat, L. Maschio, S. Casassa, M. Schütz and N. M. Harrison, Phys. Chem. Chem. Phys., 2011, 47, 4385.

15 B. Johnson and R. J. Hinde, J. Phys. Chem. A, 2011, 115, 7112.

16 C. Pisani, L. Maschio, S. Casassa, M. Halo, M. Schütz and D. Usvyat, J. Comput. Chem., 2008, 29, 2113.

17 D. Usvyat, L. Maschio, C. Pisani and M. Schütz, Z. Phys. Chem., 2010, 224, 441.

18 C. Pisani, M. Schütz, S. Casassa, D. Usvyat, L. Maschio, M. Lorenz and A. Erba, Phys. Chem. Chem. Phys., 2012, 14, 7615.

19 M. Kallay and J. Gauss, J. Chem. Phys., 2005, 123, 214105.

20 D. Usvyat, K. Sadeghian, L. Maschio and M. Schütz, Phys. Rev. B: Condens. Matter Mater. Phys., 2012, 86, 045412.

21 R. Martinez-Casado, D. Usvyat, L. Maschio, S. Casassa, J. Ellis, M. Schütz and N. M. Harrison, Phys. Rev. B: Condens.

Q4 Matter Mater. Phys., 2014, submitted.

22 F. Traeger, 2013, private communication.

23 H.-J. Werner and M. Schütz, J. Chem. Phys., 2011, 135, 144116.

24 O. Masur, D. Usvyat and M. Schütz, J. Chem. Phys., 2013, 139, 164116.

25 A. Hesselmann, G. Jansen and M. Schütz, J. Chem. Phys., 2005, 122, 014103.

26 A. J. Misquitta, R. Podeszwa, B. Jeziorski and K. Szalewicz, J. Chem. Phys., 2005, 123, 214103.

27 C. Müller, B. Herschend, K. Hermansson and B. Paulus, J. Chem. Phys., 2008, 128, 21.

28 C. Müller and K. Hermansson, Surf. Sci., 2009, 603, 3329.
29 L. Hammerschmidt, C. Müller and B. Paulus, J. Chem. Phys., 2012, 136, 124117.

30 E. Voloshina, D. Usvyat, M. Schütz, Y. Dedkov and B. Paulus, Phys. Chem. Chem. Phys., 2011, 13, 12041.

31 S. Tosoni and J. Sauer, Phys. Chem. Chem. Phys., 2010, 12,14330 .

32 A. D. Boese and J. Sauer, Phys. Chem. Chem. Phys., 2013, 15, 16481.

33 D. Usvyat, B. Civalleri, L. Maschio, R. Dovesi, C. Pisani and M. Schütz, J. Chem. Phys., 2011, 134, 214105.

34 R. Martinez-Casado, G. Mallia and N. M. Harrison, Chem. Commun., 2011, 47, 4385.

35 O. Robach, G. Renaud and A. Barbier, Surf. Sci., 1998, 401, 227.

36 R. Dovesi, V. R. Saunders, C. Roetti, R. Orlando, C. M. Zicovich-Wilson, F. Pascale, B. Civalleri, K. Doll, N. M. Harrison, I. J. Bush, P. D'Arco and M. Llunell, CRYSTAL09 User's, Università di Torino, Torino, 2010.

37 K. A. Peterson and T. H. Dunning, J. Chem. Phys., 2002, 117, 10548.

38 D. Usvyat, J. Chem. Phys., 2013, 139, 194101.

39 M. Schütz, G. Rauhut and H. J. Werner, J. Phys. Chem. A, 1998, 102, 5997.

40 See $\mathrm{ESI} \dagger$ document no. for the specification of the clusters employed.

41 H.-J. Werner, P. J. Knowles, G. Knizia, F. R. Manby and M. Schütz, Wiley Interdiscip. Rev.: Comput. Mol. Sci., 2011, 2, 242.

42 M. Schütz, G. Hetzer and H.-J. Werner, J. Chem. Phys., 1999, 111, 5691.

43 H.-J. Werner, F. R. Manby and P. J. Knowles, J. Chem. Phys., 2003, 118, 8149.

44 M. Kallay and P. Surjan, J. Chem. Phys., 2001, 115, 2945.

45 C. Müller and D. Usvyat, J. Chem. Theory Comput., 2013, 9, 5590.

46 M. Hernández, O. Roncero, S. Miret-Artés, P. Villarreal and G. Delgado-Barrio, J. Chem. Phys., 1989, 90, 3823.

47 R. Martinez-Casado, B. Meyer, S. Miret-Artes, F. Traeger and C. Woell, J. Phys.: Condens. Matter, 2007, 19, 305006.

48 R. Martinez-Casado, B. Meyer, S. Miret-Artes, F. Traeger and C. Woell, J. Phys.: Condens. Matter, 2010, 22, 304011.

49 M. Beg, Acta Crystallogr., Sect. A: Cryst. Phys., Diffr., Theor. Gen. Crystallogr., 1976, 32, 154.

50 J. Beeby, J. Phys. C: Solid State Phys., 1971, 4.

51 https://projects.ivec.org/gulp/.

52 M. Mahgefteh, D. R. Jung and D. R. Frankl, Phys. Rev. B: Condens. Matter Mater. Phys., 1989, 39, 3900.

53 A. Proctor and D. Hercules, Appl. Spectrosc., 1984, 38, 505. 